\title{
Análise da história da Revista Brasileira de Atividade Física e Saúde: 1995-2012
}

\author{
Analysis of the history of the Brazilian \\ Journal of Physical Activity and Health: \\ $1995-2012$
}

Thiago Terra Borges ${ }^{1,4,5}$

Leandro Quadro Corrêa ${ }^{2}$

Matheus Pintanel Freitas ${ }^{3,4}$

Marcelo Cozzensa da Silva $a^{3,4}$

\section{Resumo}

A Revista Brasileira de Atividade Física e Saúde (RBFS) é o periódico nacional com foco específico na relação entre atividade física e saúde. O presente estudo objetivou descrever as características das publicações na RBAFS entre os anos de 1995 e 2012. Para tanto, este estudo descritivo realizou a análise de todos os artigos contidos nos volumes da RBAFS no seu formato digital. A RBAFS publicou durante os seus 17 primeiros anos (1995-2012) 531 artigos, divididos em originais, de revisão, cartas ao editor, traduções de resumos, entrevistas e sessões especiais. A maioria das publicações foi de estudos originais $(60,4 \%)$ e o delineamento mais frequente foi o transversal $(28,3 \%)$. A temática da fisiologia do exercício concentrou o maior número de manuscritos aprovados para publicação $(18,1 \%)$ e as regiões do país que mais publicaram no periódico foram a Sudeste e Sul. Com o aumento do número de edições, a revista cria maior espaço para a publicação de pesquisas de qualidade nessa área que cresce no país a cada ano.

\section{Palavras-chave}

Revisão por Pares; História; Artigo de Jornal

\begin{abstract}
The Brazilian Journal of Physical Activity and Health (RBAFS) is the national journal focusing specifically on the relationship between physical activity and health. This study aimed to describe the characteristics of the RBAFS publications between the years 1995 and 2012. Therefore, this study conducted a descriptive analysis of all the papers published in the volumes of the RBAFS in its digital format. The RBAFS published during its first 17 years (1995-2012) 531 papers, divided into originals, reviews, letters to the editor, summaries translation, interviews and special sessions. Most publications were original studies (60.4\%) and the cross-sectional design was the most frequent (28.3\%). The exercise physiology subject had the largest number of manuscripts accepted for publication (18.1\%) and the Brazilian's regions that most published in the journal were the Southeast and South. With the increasing number of issues, the journal creates more space to the publication of quality research in this area that grows in the country each year.
\end{abstract}

\section{Keywords}

Peer review; History; Newspaper Article

Rev Bras Ativ Fis Saúde p. 371-377 $\mathrm{DO}$ :

http://dx.doi.org/10.12820/rbafs.v.18n3p371

1 Instituto Federal Sul-Rio-Grandense Campus Pelotas- Pelotas- Rio Grande do Sul- Brasil

2 Universidade Federal do Rio Grande Curso de Educação Física- Rio Grande- Rio Grande do Sul-Brasil

3 Universidade Federal de Pelotas- Programa de Pós-graduação em Educação FísicaPelotas- Rio Grande do Sul- Brasil

4 Grupo de Estudos em Epidemiologia da Atividade Física (GEEAF/UFPel)

5 Universidade Católica de PelotasPrograma de Pós-graduação em Saúde e Comportamento. 


\section{INTRODUÇÃO}

As sociedades científicas, de um modo geral, e aquelas relacionadas a área da saúde em particular, vem contribuindo, através de seus periódicos oficiais sobremaneira com a divulgação de conhecimentos associados a inúmeros desfechos em saúde, incluindo os de ordem comportamental. Por outro lado, o estreitamento das relações entre as sociedades (e seus periódicos) e os programas de pós-graduação stricto-sensu determinou a melhora na qualidade de editoração científica de modo que os artigos publicados conferem prestígio aos seus autores ${ }^{1}$.

Neste sentido, a Revista Brasileira de Atividade Física \& Saúde (RBAFS) é o periódico oficial da Sociedade Brasileira de Atividade Física \& Saúde (SBAFS), uma sociedade científica sem fins lucrativos. A RBAFS publica artigos originais, artigos de revisão, cartas ao editor, comentários, perfis e editoriais na temática da atividade física e saúde. Sendo um periódico multidisciplinar, congrega publicações de pesquisadores do campo da Educação Física, Saúde Coletiva, Nutrição, Medicina, Fisioterapia, Psicologia e áreas afins.

O periódico foi criado pela Associação dos Professores de Educação Física de Londrina em 1995 e, em janeiro de 2008, a Sociedade Brasileira de Atividade Física \& Saúde assumiu a coordenação da revista. Todos os volumes podem ser acessados na página da SBAFS (www.sbafs.org.br) ou através da plataforma eletrônica (http://periodicos.ufpel.edu.br/ojs2/index.php/RBAFS/index).

A RBAFS é o único periódico nacional com foco específico na relação entre atividade física e saúde, um tema que vem sendo cada vez mais pesquisado no mundo. Além disso, atua como parceira do Journal of Physical Activity and Health, o principal periódico internacional que publica trabalhos na área.

Desta forma, o objetivo do presente estudo foi descrever as características das publicações na RBAFS entre os anos de 1995 e 2012.

\section{METODOLOGIA}

Este estudo descritivo foi realizado através da análise de todos artigos contidos nos volumes da Revista Brasileira de Atividade Física e Saúde no período entre 1995 e 2012. Para tal, todos os volumes foram revisados através de seu formato digital disponível na plataforma, onde se encontra a versão eletrônica da revista.

Os trabalhos foram revisados por dois indivíduos com formação acadêmica na área de Educação Física, que realizaram leitura preliminar dos resumos e, quando necessário, acessaram a metodologia dos estudos para determinar o delineamento (quase-experimental, revisão, relato de experiência, ponto de vista, descritivo, transversal, experimental, validação, monografias-dissertações-teses, longitudinal, resumo, editorial, diagnóstico a ação, programas de pós graduação, intervenção e outros), o tipo de artigo (original, especial, revisão, editorial), idioma da publicação (português, inglês, traduzido do português para o inglês) e a área de abrangência do estudo (fisiologia do exercício, consequência da prática de atividade física, medidas e avaliação em atividade física e saúde, mensuração da atividade física, nível de atividade física e fatores associados, programas de pós-graduação na área 21, determinantes da prática de atividades físicas, biomecânica, experiências em promoção da atividade física, revista brasileira de atividade física e saúde, saúde pública, produção acadêmica e outros).

Os dados foram tabulados inicialmente no programa Excel e posteriormente transferidos para o programa estatístico Stata 12.0 onde foram realizadas as análises utilizando-se elementos da estatística descritiva. 


\section{RESULTADOS}

A RBAFS publicou durante os seus primeiros 17 anos (1995-2012) 531 artigos, divididos em originais, de revisão, cartas ao editor, traduções de resumos, entrevistas, e as sessões especiais como: Do Diagnóstico a Ação: Experiências em Promoção de Atividade Física e Programa de Pós-graduação da área 21. Além dos volumes publicados, algumas edições saíram com os anais do Congresso Brasileiro de Educação Física e Saúde (2007-2 e 2005-1).

A Tabela 1 apresenta, na sua primeira coluna, dados da fundação da revista até o ano de 2007. Após esta coluna a divisão se dá em biênios, com exceção da coluna equivalente ao ano de 2012, seguindo o intervalo de tempo existente entre o Congresso Brasileiro de Atividade Física e Saúde, evento este da SBAFS a qual a RBAFS está vinculada.

A maioria das publicações na RBAFS foi de estudos originais $(60,4 \%)$ seguido de artigos inseridos nas diferentes sessões especiais $(22,1 \%)$. Os delineamentos mais frequentes foram o transversal e o de revisão, respectivamente com $28,3 \%$ e 10,6\% (Tabela 1).

Tabela 1 - Descrição do tipo de artigo, delineamento dos estudos publicados na RBAFS, 1995-2012.

\begin{tabular}{lccccc}
\hline \multicolumn{1}{c}{ Variável } & $\begin{array}{c}1995-2007 \\
N(\%)\end{array}$ & $\begin{array}{c}2008-9 \\
N(\%)\end{array}$ & $\begin{array}{c}2010-11 \\
N(\%)\end{array}$ & $\begin{array}{c}2012 \\
N(\%)\end{array}$ & $\begin{array}{c}\text { Total } \\
\text { Tipo de artigo }\end{array}$ \\
\hline Original & $158(57,3)$ & $34(64,2)$ & $71(61,8)$ & $58(66,7)$ & $321(60,4)$ \\
\hline Especial & $79(28,5)$ & $5(9,4)$ & $22(19,1)$ & $11(12,6)$ & $114(22,1)$ \\
\hline Revisão & $38(13,8)$ & $2(3,8)$ & $9(7,8)$ & $6(6,9)$ & $55(10,4)$ \\
\hline Editorial & $1(0,4)$ & $12(22,6)$ & $13(11,3)$ & $12(13,8)$ & $38(7,1)$ \\
\hline Delineamento do estudo & & & & \\
\hline Transversal & $67(24,3)$ & $18(66,1)$ & $40(70,3)$ & $25(28,7)$ & $150(28,3)$ \\
\hline Revisão & $38(13,8)$ & $2(6,7)$ & $9(14,8)$ & $7(8,1)$ & $56(10,6)$ \\
\hline Descritivo & $13(4,7)$ & $6(23,0)$ & $9(14,8)$ & $15(17,2)$ & $43(8,1)$ \\
\hline Experimental & $30(10,9)$ & $0(0,0)$ & $6(10,2)$ & $6(6,9)$ & $42(7,9)$ \\
\hline Editorial & $1(0,4)$ & $12(46,1)$ & $13(23,7)$ & $12(13,8)$ & $38(7,2)$ \\
\hline Quase-experimental & $24(8,7)$ & $4(16,4)$ & $4(7,5)$ & $0(0,0)$ & $32(6,0)$ \\
\hline Monografia, dissertação e tese & $30(10,9)$ & $0(0,0)$ & $0(0,0)$ & $0(0,0)$ & $30(5,5)$ \\
\hline Validação & $16(5,8)$ & $4(15,4)$ & $7(12,2)$ & $1(1,2)$ & $28(5,3)$ \\
\hline Outros* & $13(4,5)$ & $1(4,4)$ & $7(12,2)$ & $4(4,6)$ & $25(4,7)$ \\
\hline Diagnóstico a ação & $0(0,0)$ & $5(17,7)$ & $14(24,8)$ & $4(4,6)$ & $23(4,3)$ \\
\hline Ponto de vista & $15(5,4)$ & $0(0,0)$ & $0(0,0)$ & $1(1,5)$ & $16(2,9)$ \\
\hline Longitudinal & $9(3,3)$ & $1(4,4)$ & $2(3,1)$ & $2(2,3)$ & $14(2,6)$ \\
\hline Relato de experiência & $11(4,0)$ & $0(0,0)$ & $0(0,0)$ & $0(0,0)$ & $11(2,0)$ \\
\hline PPG & $0(0,0)$ & $0(0,0)$ & $4(0,0)$ & $5(5,8)$ & $9(1,7)$ \\
\hline Resumo & $9(3,3)$ & $0(0,0)$ & $0(0,0)$ & $0(0,0)$ & $9(1,7)$ \\
\hline Intervenção & $0(0,0)$ & $0(0,0)$ & $0(0,0)$ & $5(5,8)$ & $5(0,9)$ \\
\hline
\end{tabular}

* Outros se referem a artigos do tipo especial, entrevista, tradução, preparo de resumos, ensaio, coorte, caso controle, estudo de caso, carta ao editor, censitário, ecológico, homenagem e grupo focal.

A tabela 2 descreve a área de abrangência dos estudos publicados no periódico durante o período avaliado. A temática da fisiologia do exercício $(18,1 \%)$ concentrou o maior número de manuscritos aprovados para publicação, seguida das consequências da prática de atividade física $(16,4 \%)$. 
Tabela 2 - Descrição da área de abrangência dos estudos publicados na RBAFS, 1995-2012.

\begin{tabular}{|c|c|c|c|c|c|}
\hline Variável & $\begin{array}{c}1995-2007 \\
N(\%)\end{array}$ & $\begin{array}{c}2008-9 \\
N(\%)\end{array}$ & $\begin{array}{c}2010-11 \\
N(\%)\end{array}$ & $\begin{array}{l}2012 \\
N(\%)\end{array}$ & $\begin{array}{l}\text { Total } \\
\mathrm{N}(\%)\end{array}$ \\
\hline \multicolumn{6}{|l|}{ Área de abrangência do artigo } \\
\hline Fisiologia do Exercício & $77(28,0)$ & $3(12,0)$ & $5(8,2)$ & $11(12,6)$ & $96(18,1)$ \\
\hline $\begin{array}{l}\text { Consequência da Prática de } \\
\text { Atividade Física }\end{array}$ & $52(18,9)$ & $6(23,0)$ & $20(34,9)$ & $9(10,3)$ & $87(16,4)$ \\
\hline $\begin{array}{l}\text { Medidas e Avaliação em } \\
\text { Atividade Física e Saúde }\end{array}$ & $50(18,2)$ & $4(15,4)$ & $4(6,2)$ & $3(3,5)$ & $61(11,5)$ \\
\hline $\begin{array}{l}\text { Nível de Atividade Física e } \\
\text { Fatores Associados }\end{array}$ & $24(8,7)$ & $6(22,0)$ & $13(23,7)$ & $11(12,6)$ & $54(10,2)$ \\
\hline Outros* & $26(9,4)$ & $4(15,4)$ & $6(10,6)$ & $5(5,8)$ & $41(7,7)$ \\
\hline $\begin{array}{l}\text { Mensuração da Atividade } \\
\text { Física }\end{array}$ & $14(5,1)$ & $3(10,0)$ & $17(28,0)$ & $3(3,5)$ & $37(7,0)$ \\
\hline Saúde Pública & $0(0,0)$ & $8(29,7)$ & $12(20,3)$ & $11(12,6)$ & $31(5,9)$ \\
\hline $\begin{array}{l}\text { Determinantes da Prática de } \\
\text { Atividade Física }\end{array}$ & $6(2,2)$ & $7(27,4)$ & $12(22,2)$ & $6(6,9)$ & $31(5,9)$ \\
\hline $\begin{array}{l}\text { Experiências em Promoção de } \\
\text { Atividade Física }\end{array}$ & $1(0,4)$ & $5(17,7)$ & $14(24,3)$ & $8(9,2)$ & $28(5,3)$ \\
\hline RBAFS & $1(0,4)$ & $5(19,7)$ & $4(7,5)$ & $4(4,6)$ & $14(2,6)$ \\
\hline Intervenção & $12(4,3)$ & $1(4,4)$ & $2(4,0)$ & $0(0,0)$ & $15(2,8)$ \\
\hline Biomecânica & $11(4,0)$ & $0(0,0)$ & $0(0,0)$ & $3(3,5)$ & $14(2,6)$ \\
\hline $\begin{array}{l}\text { Programas de Pós-Graduação } \\
\text { na Área } 21\end{array}$ & $1(0,4)$ & $1(3,3)$ & $5(8,6)$ & $5(5,8)$ & $12(2,3)$ \\
\hline Produção Acadêmica & $0(0,0)$ & $0(0,0)$ & $1(1,5)$ & $8(9,2)$ & $9(1,7)$ \\
\hline
\end{tabular}

* Outros se referem a artigos com temáticas de AF e saúde, antropologia, estatística, epistemologia, filosofia, currículo, conhecimento, fonoaudiologia, aprendizagem motora, comportamento, ginástica, inclusão de deficientes, universidade, capacidades física, CBAFS, saúde do trabalhador, SBAFS, ambiente, e homenagem.

Pode-se perceber na figura 1 que, após o seu surgimento em 1995, o número de artigos publicados na RBAFS aumentou até o ano de 1997 se mantendo praticamente constante até 2001. A partir deste período, houve uma queda no número de publicações até o ano de 2006, mantendo-se praticamente constante até 2008, período a partir do qual o número de artigos publicados passou a crescer significativamente.

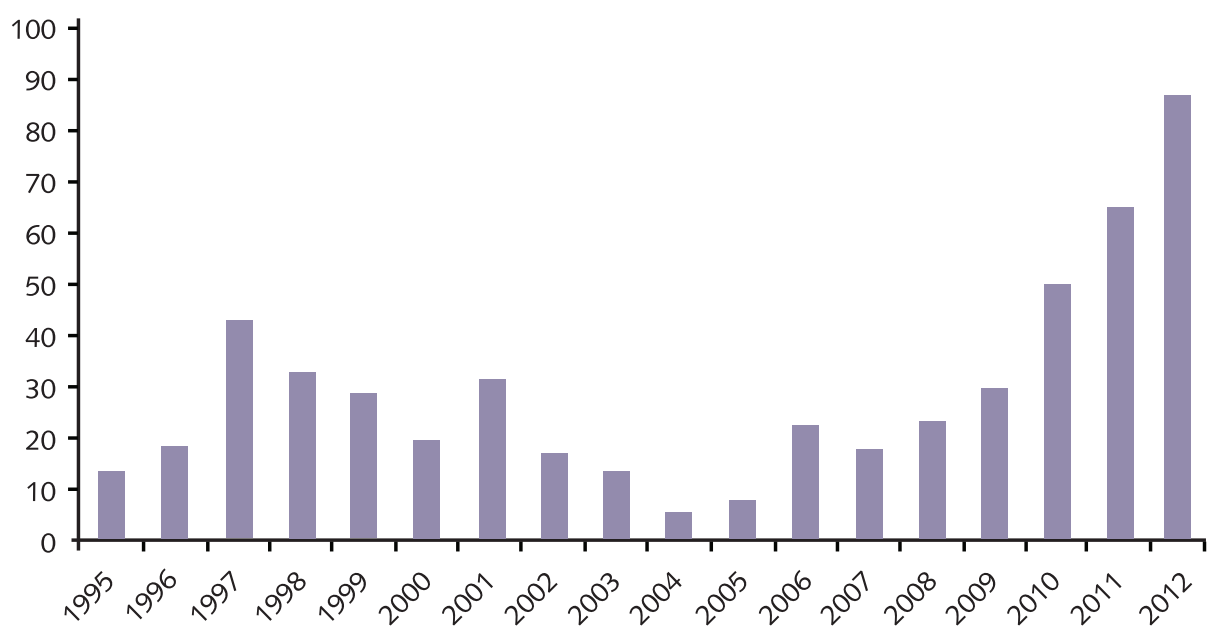

Figura 1 - Número de artigos publicados por ano na RBAFS, 1995-2012.

Desde a sua criação até o número 6 de 2012, a RBAFS publicou 518 artigos no idioma português e 13 no idioma inglês. Além disso, 15 artigos já foram traduzidos e publicados nos dois idiomas desde 2012. 
Em relação a quantidade de publicações na RBAFS, os estados das regiões $\mathrm{Su}-$ deste e Sul tiveram um maior número (Figura 2). No que diz respeito ao primeiro autor dos artigos, o estado de São Paulo é o que apresenta maior número com $34,5 \%$ do total de artigos publicados na RBAFS, seguido de Santa Catarina e Rio Grande do Sul com $17,9 \%$ e $14,9 \%$, respectivamente.

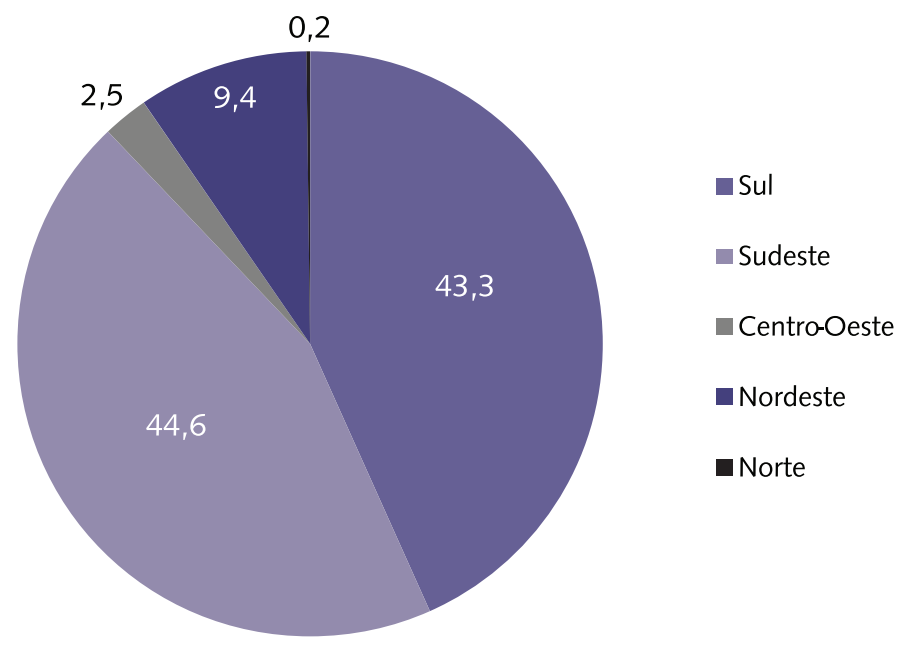

Figura 2 - Percentual de artigos publicados na RBAFS entre os anos de 1995 e 2012 de acordo com a região do país do primeiro autor.

\section{DISCUSSÃO}

Mais de um terço da produção científica brasileira a partir de 2009 foi publicada em periódicos científicos nacionais segundo os índices bibliográficos Web of Sciences (WoS) e Scopus. Tal fato ajudou o país a ocupar a $13^{a}$ posição no ranking internacional de produção científica medido pelo número de artigos publicados. Além disso, ao mesmo tempo, em 2010, aproximadamente 10,6 milhões/mês de downloads de artigos ocorreu de forma online na Scientific Electronic Library Online (SciELO) ${ }^{2}$. Apesar da RBAFS ainda não estar indexada em alguma dessas bases (a mesma, até o presente momento, está indexada na base LILACS), parece receber uma grande demanda de artigos originais. Isso é resultado do crescimento conquistado durante os últimos anos, seja em termos da quantidade de números por volume publicado, seja por ser o periódico que tem como foco principal a publicação de artigos na área de atividade física e saúde, uma demanda que estava reprimida há alguns anos, mas que, atualmente, vem crescendo exponencialmente no país devido o surgimento de cursos de pós-graduação com linhas de pesquisa em atividade física e saúde.

Apesar de, em muitos casos, apresentar dificuldade em determinar a ordem de acontecimento dos eventos e de sofrer interferência nas suas propriedades analíticas pela duração da exposição nos indivíduos com o desfecho em estudo ${ }^{3,4}$, o delineamento transversal ainda é o mais utilizado entre os estudos publicados na revista. Sua capacidade de inferência dos achados para uma população definida no tempo e espaço ${ }^{4}$ aliado ao curto período de coleta dos dados comparado a outros tipos de delineamento (coorte, caso-controle), ajuda na redução dos custos operacionais da pesquisa, fazem do mesmo uma opção de escolha muito desejada pelos investigadores.

Os dados da presente revisão indicam o avanço das pesquisas em atividade física e saúde no Brasil. No entanto, é preocupante a desigualdade de publicações quando se comparam as regiões Sudeste e Sul com as demais regiões do país. 
Estudo de Hallal e colaboradores ${ }^{5}$ sobre a evolução da pesquisa epidemiológica brasileira em atividade física já identificava essa diferença. Alguns aspectos podem ser destacados para essa discrepância encontrada. A maior quantidade de programas de pós-graduação que dispõe da linha de pesquisa em atividade física e saúde é encontrada nas regiões Sul e Sudeste ${ }^{5}$. Além disso, grupos de pesquisa bem consolidados nessa área, tais como GEEAF (Pelotas), GPAQ(Curitiba), GEPAF e CELAFISCS (São Paulo), GEPAFE (Londrina) e NuPAF (Florianópolis), ajudaram a alavancar grandemente a concentração regional das publicações. Outro fator que pode ser destacado é o financiamento das agências estaduais de pesquisa, especialmente a FAPESP (São Paulo) e a Fundação Araucária (Paraná), pela quantidade de recursos investidos em editais específicos a editoração de periódicos científicos.

O aumento do número de artigos publicados na RBAFS tornou-se notório a partir de 2007. Um dos fatores que levaram ao avanço no número de artigos submetidos e publicados se deu pela expansão da área e dos profissionais de educação física para os cursos de pós-graduação na área da saúde, seja no papel de alunos ou como docentes ${ }^{5,6}$. Outro fator que pode ter contribuído para esse aumento é que, a partir dessa data, a SBAFS passou a administrar a revista, dando um suporte mais consistente ao colocá-la como uma das prioridades da Sociedade. A demanda de submissões proporcionou o incremento do número de volumes da revista por ano. Até o ano de 2009 a revista possuía publicação quadrimestral, passando para trimestral em 2010 e bimestral em 2012.

A fisiologia do exercício no Brasil data da década de 1970, inicialmente com Prof. Maurício Leal Rocha no Laboratório de Fisiologia do Exercício da atual Universidade Federal do Rio de Janeiro e posteriormente com o grupo de pesquisas do Centro Integrado de Pesquisa em Educação Física da Universidade de São Paulo, o qual publicou os primeiros trabalhos científicos nessa década ${ }^{7}$. O surgimento de vários laboratórios e grupos de pesquisa nessa área nos anos seguintes gerou a grande demanda que sustentou os volumes iniciais da RBAFS até o ano de 2007. O aumento da quantidade de cursos Stricto-sensu com linhas de pesquisa em atividade física e saúde no Brasil a partir dos anos 2000 e o consequente aumento da produção de artigos nessa área, nas temáticas prática de atividade física, medidas e avaliação em atividade física e saúde e nível de atividade física e fatores associados, entre outros, levou a revista em 2008 a redefinir seu escopo, de modo a privilegiar a publicação de artigos que trouxessem contribuições para o desenvolvimento da área de "atividade física e saúde".

A RBAFS vem experimentando grande crescimento nos últimos anos. A divulgação do periódico junto aos cursos de graduação e pós-graduação no Brasil, o aumento da produção científica específica na área e a criação de sessões especiais como "Do diagnóstico a ação: Experiência em promoção da saúde" e "Programas de Pós-Graduação na área 21" parecem ser fatores que ajudaram a aumentar consideravelmente o número de submissões na mesma.

\section{Contribuição dos autores}

Thiago Terra Borges e Leandro Quadro Corrêa idealizaram o estudo e lideraram a escrita do artigo e análise dos dados. Matheus Pintanel Freitas participou da escrita do artigo e da análise dos dados. Marcelo Cozzensa da Silva idealizou o estudo e participou de todas as etapas de construção deste trabalho. 


\section{REFERÊNCIAS}

1. Cuenca AMB, Noronha DP, Ueno HM, Kobayashi KM. Periódicos brasileiros em saúde pública: a questão do financiamento. InCID: Rev Cie Inf Doc. 2011;2(2):101-10.

2. Packer AL. Os periódicos brasileiros e a comunicação da pesquisa nacional. Rev. USP [online]. 2011;89:26-61.

3. Rothman KJ, Greenland S, Lash TL. Epidemiologia Moderna. 3a ed. Porto Alegre: Artmed, 2011.

4. Medronho RA. Epidemiologia. São Paulo: Atheneu, 2005.

5. Hallal PC, Dumith SC, Bastos JP, Reichert FF, Siqueira FV, Azevedo MR. Evolução da pesquisa epidemiológica em atividade física no Brasil: revisão sistemática. Rev. Saúde Pública [online]. 2007;41(3):453-60.

6. Rigo LC, Ribeiro GM, Hallal PC. Unidade na diversidade: desafios para a Educação Física no século XXI. Rev Bras Ativ Fis e Saude. 2001;16(4):339-45.

7. Forjaz CLM, Tricoli V. A fisiologia em educação física e esporte. Rev. Bras. Educ. Fís. Esporte [online]. 2011;25:7-13.

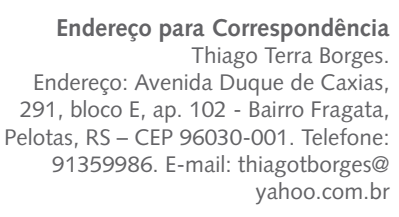

Recebido 17/08/2013

Revisado 09/09/2013

Aprovado 10/09/2013 